\title{
The MYB transcription factor CiMYB42 regulates limonoids biosynthesis in citrus
}

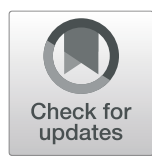

Pan Zhang ${ }^{1,2}$, Xiaofeng Liu ${ }^{1,2}$, Xin Y Y ${ }^{1,2}$, Fusheng Wang ${ }^{1,2}$, Junhong Long ${ }^{1,2}$, Wanxia Shen ${ }^{1,2}$, Dong Jiang ${ }^{1,2}$ and Xiaochun Zhao ${ }^{1,2^{*}}$

\begin{abstract}
Background: Limonoids are major bioactive compounds that are produced by the triterpenoid metabolic pathway. The detailed biochemical process of limonoid biosynthesis and the mechanism of its molecular regulation remain elusive. The identification of transcription factors that regulate limonoid biosynthetic pathways is very important for understanding the underlying regulatory mechanisms. This information could also provide tools for manipulating biosynthesis genes to modulate limonoid production.
\end{abstract}

Results: In this study, the CiMYB42 transcription factor was isolated to identify its role in limonoid biosynthesis. Multiple alignment analysis and phylogenetic analysis demonstrated that CiMYB42 is a typical R2R3MYB transcription factor that shares high similarity of its amino acid sequence with AtMYB42. Limonoids contents were higher in Citrus sinensis and Citrus grandis than in other species. Limonoid accumulation during leaf development also showed diverse trends in different genotypes. The expression of CiMYB42 was significantly related to the limonoid content and the expression of CIOSC in some citrus accessions. The overexpression of CiMYB42 in sweet orange resulted in significant accumulation of limonin, whereas the downregulation of CiMYB42 by RNAi resulted in a dwarf phenotype and less nomilin accumulation. Furthermore, the results of a yeast one-hybrid assay and EMSA indicated that CiMYB42 binds exclusively to the TTGTTG sequence (type II MYB core) in the promoter of CiOSC. Together, these results suggest that CIMYB42 positively regulates limonoid biosynthesis by regulating the expression of CiOSC by binding to the TTGTG sequence (type II MYB core) of its promoter.

Conclusions: CIMYB42 is an important transcription activator involved in limonoid biosynthesis that regulates the expression of CiOSC by binding to the TTGTG sequence (type II MYB core).

Keywords: Limonoid biosynthesis, R2R3MYB, CiMYB42, CIOSC, Triterpenoid

\section{Background}

Citrus is one of the most important fruit crops in the world. Citrus produces diverse secondary metabolites, including limonoids. Limonoids possess extensive biological and pharmacological activities [1], such as antioxidant [2] and insect antifeedant [3, 4] as well as antibacterial $[5,6]$, anticancer [7-9], antiviral [10, 11],

\footnotetext{
* Correspondence: zhaoxiaochun@cric.cn

${ }^{1}$ Citrus Research Institute, Southwest University/Chinese Academy of Agricultural Sciences, Beibei, Chongqing 400712, China

${ }^{2}$ National Citrus Engineering Research Center, Beibei, Chongqing 400712, China
}

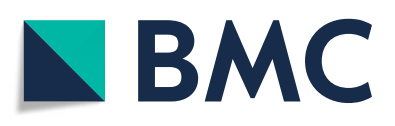

(c) The Author(s). 2020 Open Access This article is licensed under a Creative Commons Attribution 4.0 International License, which permits use, sharing, adaptation, distribution and reproduction in any medium or format, as long as you give appropriate credit to the original author(s) and the source, provide a link to the Creative Commons licence, and indicate if changes were made. The images or other third party material in this article are included in the article's Creative Commons licence, unless indicated otherwise in a credit line to the material. If material is not included in the article's Creative Commons licence and your intended use is not permitted by statutory regulation or exceeds the permitted use, you will need to obtain permission directly from the copyright holder. To view a copy of this licence, visit http://creativecommons.org/licenses/by/4.0/ The Creative Commons Public Domain Dedication waiver (http://creativecommons.org/publicdomain/zero/1.0/) applies to the data made available in this article, unless otherwise stated in a credit line to the data.

and anti-inflammatory [12] activities. The production of limonoids varies in different citrus species, organs and tissues, and developmental stages [13-15].

Limonoids are tetracyclic triterpene compounds that are synthesized from isopentenyl diphosphate (IPP) and dimethylallyl diphosphate (DMAPP) via the mevalonate (MVA) pathway and methylerythritol phosphate (MEP) pathway, respectively $[16,17]$. The condensation of IPP and DMAPP forms C15 farnesyl diphosphate (FPP), which is further transformed into the linear C30 triterpenoid precursor squalene catalysed by squalene synthase (SQS) in a head-to-head condensation reaction. 
Subsequently, squalene epoxidase (SQE) oxidizes squalene to form 2,3-oxidosqualene, which undergoes cyclization mediated by specific oxidosqualene cyclases (OSCs) to form diverse triterpenoid skeletons [18]. A schematic diagram of limonoid biosynthesis is shown in Fig. S1. Squalene is the first precursor of triterpenoids such as limonoids, sterols, and brassinosteroids. SQS plays an important regulatory role in triterpenoid biosynthesis because it is located at a key branch point and acts as a switch [19]. SQE and OSC are the key ratelimiting enzymes in triterpenoid biosynthesis, catalysing the first oxygenation and cyclization steps, respectively $[20,21]$. Strategies for altering triterpenoid production by manipulating genes that encode triterpenoid pathway enzymes have been reported [22-25]. Transcription factors (TFs) present great potential for improving the production of secondary metabolites by activating or repressing structural genes in metabolic pathways by binding to their promoter regions [26]. Hence, they are ideal targets for genetically manipulating the production of triterpenoids.

None of the TFs found in citrus have been reported to regulate triterpenoid production, but several TFs from other plants involved in triterpenoid biosynthesis have been identified. Shang et al. [27] reported that the $B l$ (bitter leaf) and $B t$ (bitter fruit) bHLH TFs regulate the biosynthesis of cucurbitacin $C$ by binding to the promoter of $B i$ (a member of OSC) in cucumber (Cucumis sativus). TSAR1 (TRITERPENE SAPONIN BIOSYNTHESIS ACTIVATING REGULATOR1) and TSAR2 are two homologous jasmonate-inducible bHLH transcription factors that directly influence triterpene saponin biosynthesis by interacting with the promoters of HMGR1 (3-HYDROXY-3-METHYLGLUTARYLCOENZYME A REDUCTASE1) and MAKIBISHI1 in Medicago truncatula [28]. The liquorice (Glycyrrhiza uralensis) bHLH TF GubHLH3 positively regulates the expression of triterpenoid saponin biosynthetic genes [29]. Recently, WsWRKY1 of Withania somnifera was found to directly regulate the triterpenoid pathway by binding to W-box sequences in the promoters of $S Q S$ and $S Q E$ [30]. In addition, MYB TFs are crucial regulators that participate in plant terpenoid metabolism. The overexpression of grapevine (Vitis vinifera) VvMYB5b in tomato induced interesting effects, including the downregulation of phenylpropanoid metabolism and beta-amyrin and upregulation of beta-carotene was up regulated [31]. P. taeda PtMYB14 is also related to terpenoid biosynthesis [32, 33]. Both PtMYB14 and $V v M Y B 5 b$ are members of $R 2 R 3-M Y B$ s, which are likely to regulate terpenoid biosynthesis. Recently, another R2R3-MYB member, SmMYB36 from Salvia miltiorrhiza Bunge, has been reported to promote the accumulation of diterpenoids (tanshinone) [34].
$R 2 R 3 M Y B$ transcription factors are one of the largest families of plant TFs. The members of this family extensively participate in terpenoid biosynthesis, not only that of triterpenoids but also those of other terpenoids. For example, spearmint (Mentha spicata) MsMYB can bind to the cis-elements of MsGPPS. LSU and suppress monoterpene biosynthesis [35]. Artemisia annua AaMYB1 acts as an activator in diterpene metabolism (artemisinin, AN) [36]. Despite the identification of R2R3MYBs in plant terpenoid biosynthesis, their roles in triterpenoid metabolism are still poorly understood, especially in limonoid biosynthesis.

In our previous study, ciclev10021695m, an MYB family TF, was revealed to be related to the biosynthesis of limonoids by RNA-seq analysis [20]. In this study, the role of CiMYB42 was investigated to elucidate the regulatory mechanism of CiMYB42 in the biosynthesis of limonoids in citrus.

\section{Results \\ Characteristics of CiMYB42}

The genomic sequence of ciclev $10021695 \mathrm{~m}$ was obtained from the $C$. clementina genomic database (https://phytozome.jgi.doe.gov/pz/portal.html\#!info?alias=Org_Cclementina). It encodes 267 amino acids and has a theoretical isoelectric point and molecular weight of 5.14 and $30.21 \mathrm{kDa}$, respectively. Ciclev10021695m is an R2R3 MYB transcription factor because it contains a typical conserved R2R3 MYB domain. The amino acid sequence of ciclev10021695m shares high similarity with AtMYB42 of Arabidopsis (55.86\%), and it was accordingly designated CiMYB42 (Fig. 1a \& b).

Phylogenetic analysis of CiMYB42 with other R2R3$M Y B$ genes from Arabidopsis indicated that CiMYB42 is closely related to AtMYB42 and AtMYB85, which have been reported to be involved in plant secondary metabolism [37]. In addition, our previous work demonstrated that the expression level of CiMYB42 is significantly related to limonoid content in pummelo seeds [20]. This suggested that CiMYB42 could act as a regulatory factor in limonoid biosynthesis.

\section{Accumulation of limonoids during leaf development}

Leaf samples from nine different citrus accessions at three different developmental stages (Fig. S2) were used for the determination of limonoid (limonin and nomilin) contents in this study. The scientific names and abbreviations of nine citrus accessions were shown in Table 1. Limonoid contents varied among different accessions and leaf developmental stages (Fig. 2) and ranged from $0.02 \mathrm{mg} / \mathrm{g}$ FW to $0.53 \mathrm{mg} / \mathrm{g}$ FW in the nine citrus accessions. The highest limonoid content was 26 times greater than the lowest. Variation in limonoid contents among different citrus species was also observed. $C$. 


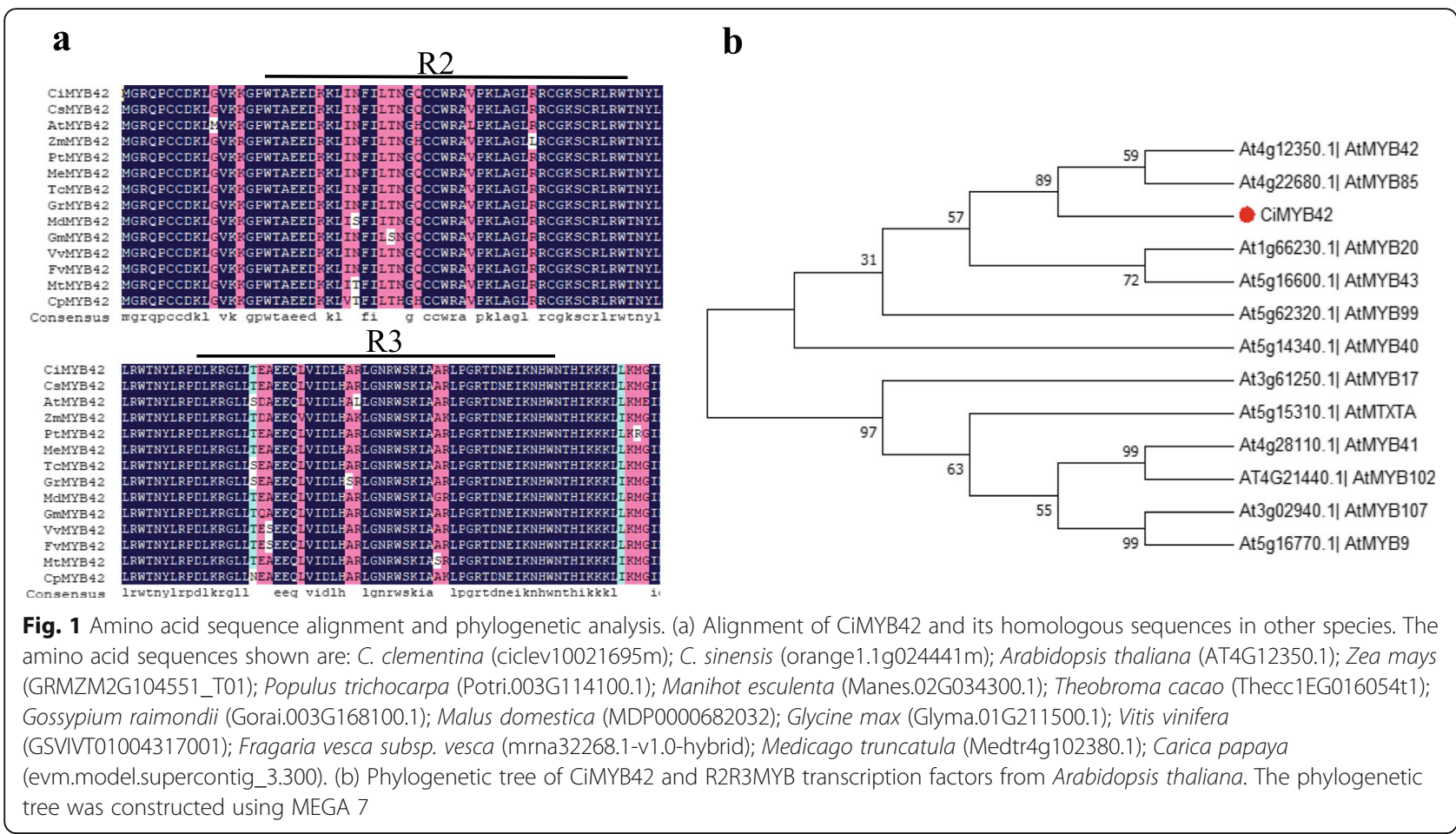

sinensis presented the highest limonoid content (0.25$0.53 \mathrm{mg} / \mathrm{g} \mathrm{FW})$, followed by C. grandis $(0.15-0.22 \mathrm{mg} / \mathrm{g}$ FW). However, limonoid contents remained at a quite low level in C. reticulata and F. classifolia, especially in F. classifolia. This revealed that the genotype is one of the most important factors influencing limonoid content. In addition, several different patterns of limonoid accumulation during leaf developmental stages were found in different accessions (Fig. 2). Specifically, limonoid content first increased and then decreased throughout the period of leaf growth and maturation in JC and ZSXLMY but changed little. The limonoid content of NHE changed the most markedly and noticeably decreased in the Y2 period. In ST, it remained at a quite low level throughout the leaf growth period, and the trend of the changes was similar to that in NHE. In
WCZPG, the limonoid content constantly increased during growth, reaching 0.04, 0.07 and $0.08 \mathrm{mg} / \mathrm{g} \mathrm{FW}$. The limonoid content showed no obvious change in the other varieties.

\section{Correlation between CiMYB42 expression and limonoid content}

The relative expression of CiMYB42 exhibited significant differences among the accessions and leaf developmental stages (Fig. 3). The highest expression level (8.17) was observed in ZSXLMY at the Y2 stage, whereas the lowest expression level (0.70) was found in the Y2 stage of GC, which presented 12-fold lower expression. The expression level of CiMYB42 showed trends similar to those of limonoid content during leaf development. Significant positive correlations between the level of

Table 1 Citrus samples used in this study

\begin{tabular}{lll}
\hline Name of species & Name of accessions & Abbreviation used in this study \\
\hline Citrus sinensis (L.) Osbeck & Beibei 447 orange & JC \\
Citrus sinensis (L.) Osbeck & Newhall navel orange & NHE \\
Citrus reticulata Blanco & Wangcang Zoupigan mandarin & WCZPG \\
Citrus reticulata Blanco & Morita Unshu mandarin & ST \\
Citrus reticulata Blanco & Miyagawa Wase mandarin & GC \\
Fortunella classifolia Swingle & Huapi kumquat & HPJG \\
Fortunella classifolia Swingle & Rongan kumquat & RAJG \\
Citrus grandis (L.) Osbeck & Guangxi Shatianyou pummelo & GXSTY \\
Citrus grandis (L.) Osbeck & Early Siam pummelo & ZSXLMY \\
\hline
\end{tabular}




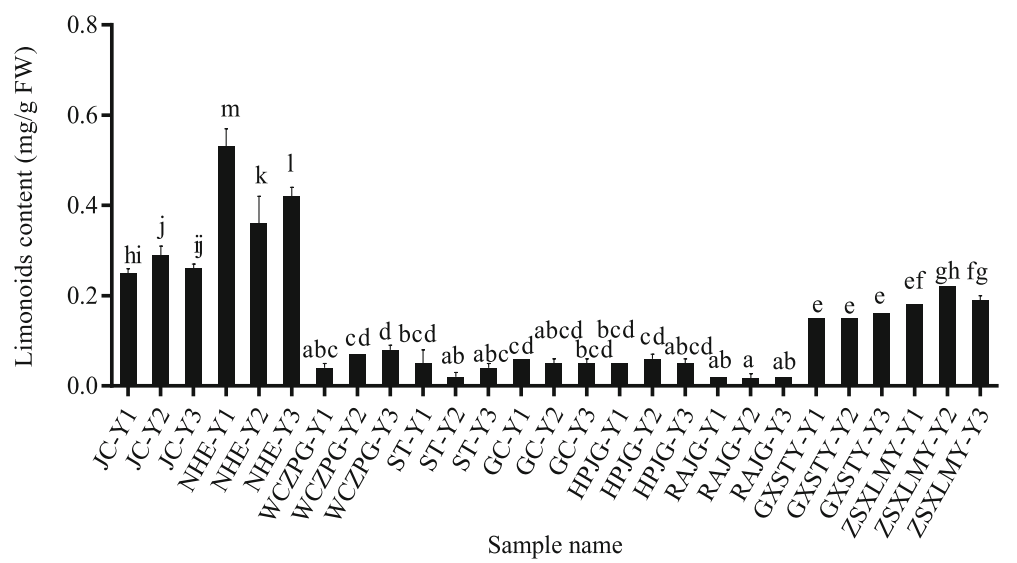

Fig. 2 Limonoids content in different citrus varieties during leaf development. The abbreviations in figure are as follows: JC, Beibei 447 orange; NHE, Newhall navel orange; WCZPG, Wangcang Zoupigan mandarin; ST, Morita Unshu Mandarin; GC, Miyagawa Wase Mandarin; HPJG, Huapi kumquat; RAJG, Rongan kumquat; GXSTY, Guangxi Shatian pummelo; ZSXLMY, Early Siam pummelo; Y1, Y2 and Y3 are three leaf development stages, respectively

CiMYB42 expression and limonoid content during leaf development were found in JC, WCZPG and GC (Table 2). However, some strong negative correlations were also observed (HPJG, GXSTY and ZSXLMY). Positive correlations between CiMYB42 expression and limonoid content were found at all three leaf developmental stages among these accessions, especially in the Y3 stages $(0.236,0.639$ and 0.66 , respectively). Similar correlations were observed between limonoid content and CiOSC expression. However, the expression of CiMYB42 presented a significant correlation with $\mathrm{CiOSC}$ during leaf development in most of the accessions, with the exception of GC. A close correlation was observed among the accessions in all three developmental stages (0.971, 0.824 and 0.81 , respectively).

\section{Effect of CiMYB42 on limonoid production in transgenic plants}

The overexpression and RNA interference knockdown of CiMYB42 were conducted to further elucidate the role of CiMYB42 in limonoid biosynthesis. The recombinant overexpression and RNA interference vectors are shown in Fig. 4a \& Fig. 4b. Non-transgenic Wanjincheng orange (C. sinensis (L.) Osbeck) was grafted onto the rootstock used as the control. Three CiMYB42-overexpressing (CiMYB42-OE) lines and three CiMYB42 RNA interference (CiMYB42-R) lines (Fig. 4c \& Fig. 4d) were obtained via Agrobacterium-mediated transformation. Images of the control and GFP-positive cells under an ultraviolet lamp are shown in Fig. 4e. R-2 transgenic plants were used as an example to provide GFP-positive

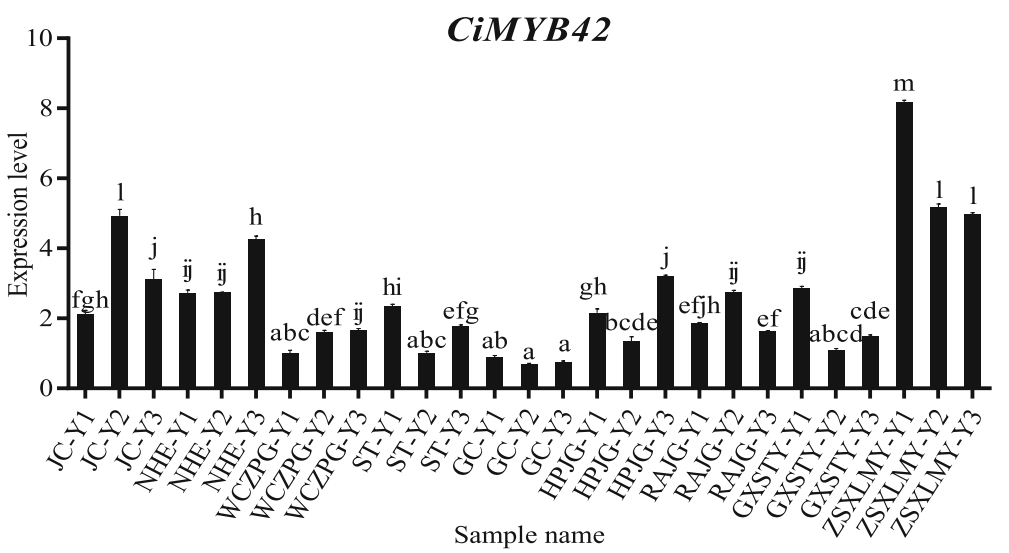

Fig. 3 CiMYB42 expression level in different citrus accessions during leaf development. The abbreviations are: JC, Beibei 447 orange; NHE, Newhall navel orange; WCZPG, Wangcang Zoupigan mandarin; ST, Morita Unshu Mandarin; GC, Miyagawa Wase Mandarin; HPJG, Huapi kumquat; RAJG, Rongan kumquat; GXSTY, Guangxi Shatian pummelo; ZSXLMY, Early Siam pummelo; Y1, Y2 and Y3 are three leaf development stages, respectively 
Table 2 Correlations between CiMYB42 expression and limonoids content

\begin{tabular}{llllllllll}
\hline Pearson Correlation Coefficient & JC & NHE & WCZPG & ST & GC & HPJG & RAJG & GXSTY & ZSXLMY \\
\hline CiMYB42\& Limonoids & $0.78^{*}$ & -0.155 & $0.835^{* *}$ & 0.615 & $0.691^{*}$ & $-0.768^{*}$ & -0.25 & $-0.781^{*}$ & $-0.666^{*}$ \\
CiOSC\& Limonoids & $0.737^{*}$ & -0.321 & $0.885^{* *}$ & 0.575 & $-0.736^{*}$ & $-0.701^{*}$ & -0.382 & -0.359 & $-0.703^{*}$ \\
CiMYB42\& CiOSC & $0.943^{* *}$ & $0.942^{* *}$ & $0.689^{*}$ & $0.963^{* *}$ & $-0.844^{* *}$ & $0.855^{* *}$ & $0.964^{* *}$ & $0.708^{* *}$ & $0.998^{* *}$ \\
\hline
\end{tabular}

${ }^{*} P<0.05 ; * * P<0.01$

images. No morphological differences were observed between the control and overexpression lines, but the RNAi lines exhibited dwarfing and shorter internode characteristics (Fig. 4d). Subsequently, real-time qPCR was performed to evaluate the expression levels of CiMYB42, CiSQS and CiOSC in the transgenic lines and controls (Fig. 4f). CiMYB42 expression was significantly higher in the overexpression lines than in the control, especially in overexpression lines 1 and 3, which showed approximately 6.6 times higher expression than the control. Three RNAi transgenic lines (R-1, R-2 and R-3) showed reduced levels of CiMYB42 expression to different extents, exhibiting expression decreases of 39,67 and $72 \%$, respectively. The expression of CiSQS was not suppressed by silencing CiMYB42 in RNAi plants and was not upregulated in the overexpression plants (Fig. 4f). The correlation between the expression of these two genes was significantly negative. In contrast, the expression of $\mathrm{CiOSC}$ was consistent with the expression of CiMYB42 in transgenic plants.

The altered expression of CiOSC and CiMYB42 altered limonoid accumulation in the overexpression and RNAi lines. The limonoid content increased by $16.08 \%$ in the overexpression lines and decreased by $22.09 \%$ in the RNAi lines (Fig. 4g). Notably, transgenic CiMYB42 had different effects on limonin and nomilin contents. The limonin content was increased by $50 \%$ in the overexpression plants, but there was no significant difference between the RNAi lines and the control. In contrast, nomilin contents exhibited a great decline in the RNAi lines, particularly in line 2 (decreased by $48.76 \%$ ). In the overexpression lines, the nomilin content only showed a slight increase (3.31\%). Thus, the overexpression of CiMYB42 mainly increased the limonin content, while CiMYB42 RNAi mostly decreased the nomilin content.

Correlation analysis indicated that limonoid contents were significantly correlated with the expression of CiMYB42 and CiOSC in the transgenic plants, with Pearson correlation coefficients of $0.824(\mathrm{P}<0.05)$ and 0.931 $(\mathrm{P}<0.01)$, respectively. However, the limonoid content was negatively correlated with the expression of CiSQS.

\section{CiMYB42 regulates limonoid biosynthesis by binding to the promoter of CiOSC}

The significant correlations of gene expression between CiMYB42, CiOSC, and CiSQS suggested the possible interaction of CiMYB42 with CiOSC or CiSQS. These
TF binding cis-elements were identified in promoters of CiOSC and CiSQS by using PLANTCARE and PLACE online software (Fig. S3). The approximately $2 \mathrm{~kb}$ promoters of CiOSC and CiSQS contained several MYB cores and $\mathrm{AC}$ elements, which are required for MYB binding [38]. On the basis of these cis-element analyses, a $\mathrm{Y} 1 \mathrm{H}$ assay was carried out to identify potential interactions between CiMYB42 and the promoters of CiOSC and CiSQS. The minimal AbA inhibitory concentration of the bait vector was detected as shown in Fig. S4. In addition to the $\mathrm{Y} 1 \mathrm{H}$ system control, the cotransformation of empty pGADT7 and pAbAi-SQS/OSC was also performed to reduce the false positive rate (Fig. $5 \mathrm{a} \& \mathrm{~b}$ ). The results indicated that CiMYB42 directly and exclusively interacted with the CiOSC promoter under suppression by $500 \mathrm{ng} / \mathrm{mL} \mathrm{AbA}$ (Fig. $5 \mathrm{~d}$ ). In contrast, there was no interaction between CiMYB42 and the CiSQS promoter in yeast cells (Fig. 5c). This suggests that CiMYB42 acts as an activator regulating the expression of CiOSC. These results indicated that CiMYB42 could be one of the key factors involved in the biosynthesis of limonoids by regulating the expression of $\mathrm{CiOSC}$.

\section{CiMYB42 mediates CiOSC transactivation by binding to the type II MYB core cis-element in the CiOSC promoter} Many studied MYB proteins act via the recognition of the MYB core sequence (C/TNGTTG/A) and AC elements (ACCA/TAA/CT/C) in promoter regions $[38,39]$. However, MYBs exhibit different affinities for these sequences. The pioneering work of Kelemen et al. [38] elucidated the preferential interaction of AtMYB85 with the $\mathrm{AC}$ element and type II MYB core, especially for the type II MYB core. Based on the sequence similarity between CiMYB42 and AtMYB42 and AtMYB85, we deduced that CiMYB42 may also interact with $\mathrm{AC}$ elements and the type II MYB core. Therefore, we performed electrophoretic mobility shift assays (EMSAs) with recombinant proteins and three biotin-labelled probes containing these cis-element sequences (Fig. 6). The expression and purification of the CiMYB42 protein are shown in Fig. S5. The original image of Figure S5 is shown in Figure S6. The original EMSA gel image is shown in Figure S7. As shown in Fig. 6, the C-terminally His-tagged CiMYB42 protein exhibited a strong affinity for the OSC-2 probe with the TTGTTG sequence but. 


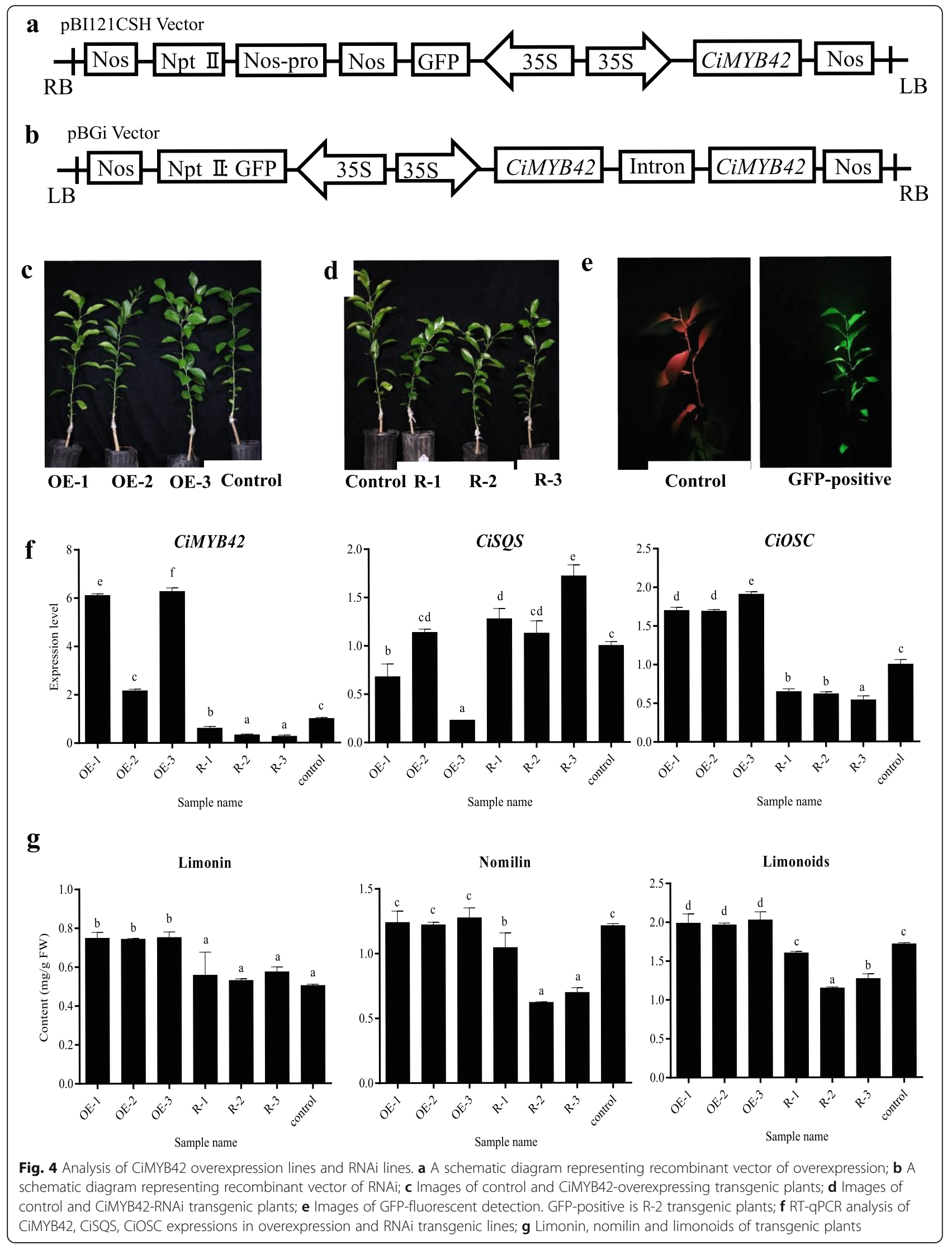



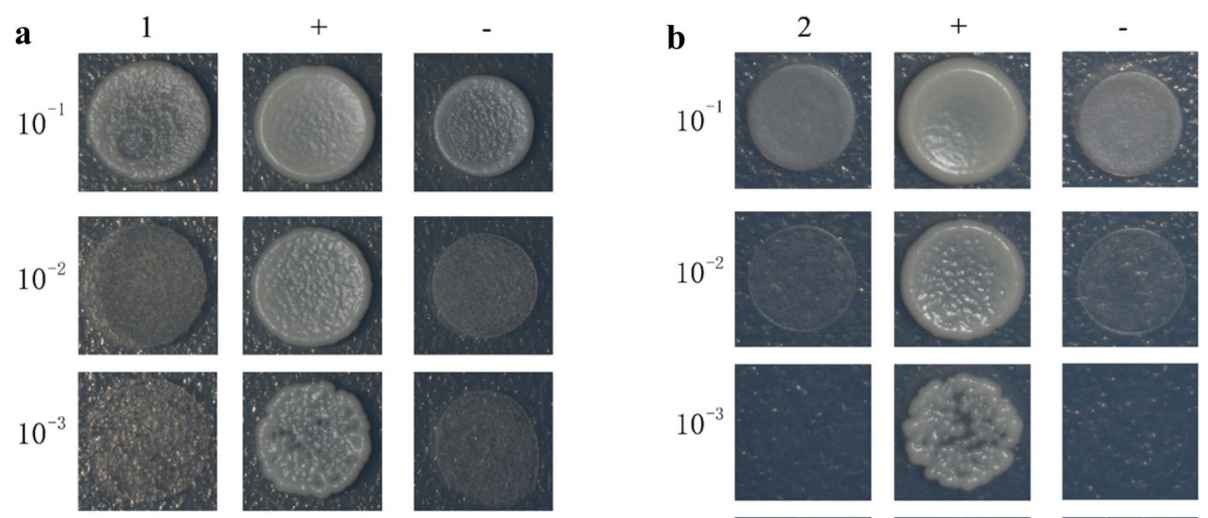

$10^{-4}$
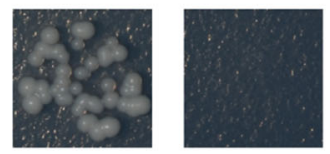

$10^{-4}$
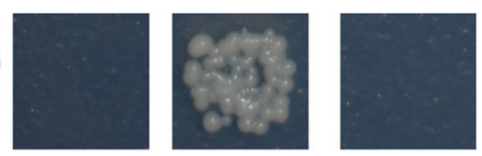

c
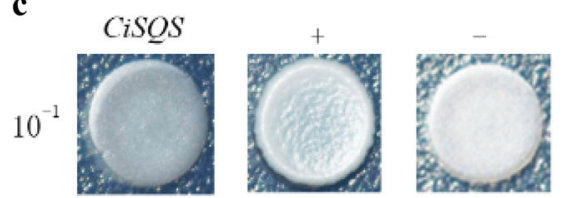

d
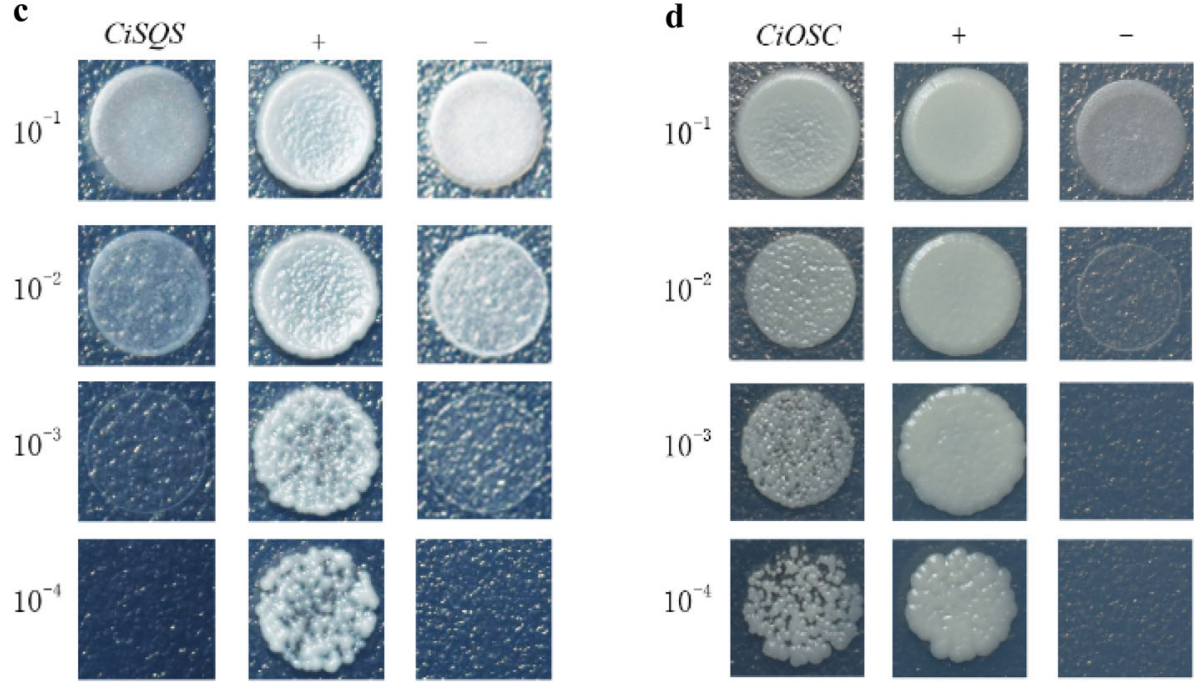

Fig. 5 Identification of interaction between CiMYB42 and promoters of CiSQS and CiOSC with yeast one-hybrid assays. a Co-transformation of empty pGADT7 and pAbAi-SQS under the condition of $200 \mathrm{ng} / \mathrm{ml} \mathrm{AbA;} \mathrm{b} \mathrm{Co-transformation} \mathrm{of} \mathrm{empty} \mathrm{pGADT7} \mathrm{and} \mathrm{pAbAi-OSC} \mathrm{under} \mathrm{the}$ condition of $500 \mathrm{ng} / \mathrm{ml} \mathrm{AbA;}$ c The interaction between CiMYB42 and CiSQS under the condition of $200 \mathrm{ng} / \mathrm{ml} \mathrm{AbA;} \mathrm{d} \mathrm{The} \mathrm{interaction} \mathrm{between}$ CiMYB42 and CiOSC under the condition of $500 \mathrm{ng} / \mathrm{ml} \mathrm{AbA}$; (+) Positive control: pGADT7-p53 + Y1H [pAbAi-p53]; (-) Negative control: pGADT7 + Y1H [pAbAi-p53]; (1) pGADT7 + Y1H [pAbAi-SQS]; (2) pGADT7 + Y1H [pAbAi-OSC]; the recombinant Y1H Gold yeast strain were selected by SD/ -leu medium containing optimal AbA concentrations; 10-1, 10-2, 10-3 and 10-4 are different dilution ratio

was not capable of binding to the OSC-1 (ACCAAAC, AC-element) and OSC-3 (TAACTA, type II MYB core) probes. These results showed that CiMYB42 functions as an R2R3-type MYB transcriptional activator, which binds to the type II MYB core (TTGTTG) sequence in the CiOSC promoter and activates the transcription of the $\mathrm{CiOSC}$ gene in citrus.

\section{Discussion}

In this study, the CiMYB42 gene was identified as significantly affecting limonoid biosynthesis by regulating the expression of CiOSC. A previous report indicated that CiMYB42 (ciclev10021695m) is an R2R3MYB gene located on scaffold S00271 of sweet orange (C. sinensis) and S3 of clementine (C. reticulata). Its expression can be induced by cold stress and ABA and JA treatments [40], but its function is unknown. In general, $R 2 R 3 M Y B$ transcription factors play important regulatory roles in terpenoid biosynthesis, especially the members in subgroups 4, 5, and 15 [33, 34]. CiMYB42 was classified in subgroup 12 in a previous report [40]. Our study suggested that members of subgroup 12 may also be involved in the regulation of terpenoid biosynthesis.

Variations in limonoid contents in different species and developmental stages of citrus seeds and fruits have been reported [13, 15, 41, 42], but there has been less 


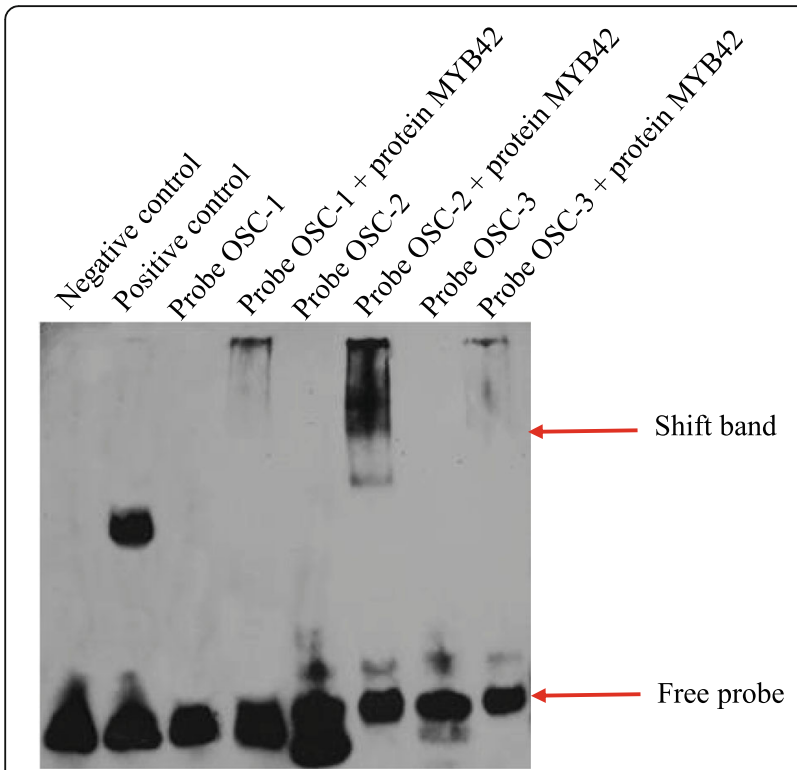

Fig. 6 EMSA of CiMYB42 binding to the fragments of the CiOSC promoter. The recombinant protein of CiMYB42 fused with $6 \times$ His tag was incubated with biotin-labeled probes and subjected to EMSA by polyacrylamide gel electrophoresis. Negative control: NF-KB probe (AGTTGAGGGGACTTTCCCAGGC); Positive control: NF-KB probe + nuclear protein of Hela cells. The gel image were cropped because original image includes some of other samples not related to this study

focus on limonoid accumulation during leaf development in different citrus species. In this study, limonoid contents were found to be significantly different among different leaf developmental stages in most of the examined accessions. The trends of limonoid accumulation during leaf development presented several different patterns depending on the genetic background. The expression of CiMYB42 was not positively correlated with the limonoid contents of some accessions during leaf development (Table 1). However, similar correlations were observed between the expression of $\mathrm{CiOSC}$ and limonoid contents, possibly due to $\mathrm{CiOSC}$ indirectly participating in the limonoid biosynthetic pathway by regulating the synthesis of triterpene [43]. Furthermore, limonoid accumulation is affected not only by the biosynthesis of limonoids but also by factors such as the transportation and degradation of limonoids.

A reverse genetics approach was applied to determine the role of CiMYB42 in limonoid biosynthesis. Some interesting phenotypes were identified in CiMYB42-silenced citrus plants (Fig. 4d). RNAi knockdown of CiMYB42 resulted in stunted growth and shorter internodes of the plants exhibiting a significant decrease in limonoid contents. The phenomena of morphogenetic inhibition and reduced limonoid production resulting from the silencing of CiMYB42 were similar to the findings of previous studies involving the downregulation/ silencing of triterpenoid pathway genes and triterpenoid metabolism-related TFs such as NtCAS1 [44], AtHMGR1 [45] and WsWRKY1 [30]. The decrease in the nomilin content induced by the RNAi knockdown of CiMYB42 could be a result of the depletion of limonoid precursors, which are required for limonoid production [46]. The downregulation of $\mathrm{CiOSC}$ expression may also resulted in nomilin reduction. However, the small change in limonin production is likely due to a sufficient nomilin supply remaining for limonin synthesis despite the dramatic decrease in nomilin. A downregulation of $\mathrm{CiOSC}$ expression was induced by the RNAi knockdown of CiMYB42 in citrus plants. However, CiSQS was slightly upregulated by CiMYB42 RNAi. Such differential regulation of triterpenoid biosynthesis has recently been reported in W. somnifera (involving WsWRKY1) [30], in tomato (involving GAME9) [47], and in birch (Betula platyphylla Suk.; involving BpbHLH9) [48].

In contrast to CiMYB42 RNAi, the overexpression of CiMYB42 in sweet orange leaves increased the expression level of $\mathrm{CiOSC}$ and limonoid contents. Furthermore, the $\mathrm{Y} 1 \mathrm{H}$ assay provided further support for the vital role of CiMYB42 based on the finding that CiMYB42 exclusively activated the promoter of CiOSC. These results are consistent with the conclusion that CiMYB42 positively regulates limonoid biosynthesis. The overexpression of CiMYB42 induced the accumulation of limonin rather than nomilin. This suggests that CiMYB42 may have other targets. In addition to $\mathrm{CiOSC}$, other downstream genes in the limonoid pathway may be induced by CiMYB42. One target gene may be regulated by several TFs; conversely, one TF may be involved in multiple biosynthetic processes [39, 49].

Previous reports showed that MYB TFs could bind to the MYB core sequence and $\mathrm{AC}$ elements $[50,51]$. The EMSA results indicated that CiMYB42 could specifically bind to the TTGTTG sequence of the CiOSC promoter (Fig. 6), suggesting that CiMYB42 preferentially binds to the type II MYB core (TNGTTG/A), similar to AtMYB85 [38]. Based on the results obtained for two close homologs of CiMYB42, AtMYB42 and AtMYB85 (Fig. 1b), suggest that $C i M Y B 42$ may also regulate secondary cell wall biosynthesis. This is the first report elucidating the role of transcription factors in Citrus limonoid biosynthesis. Our contributions will provide a reference for understanding the regulatory mechanisms of R2R3MYB TFs in the triterpenoid biosynthetic pathway.

\section{Conclusions}

In this study, we identified a novel regulatory factor, CiMYB42, that is involved in limonoid biosynthesis by binding to the type II MYB core (TNGTTG/A) sequence in the promoter of $\mathrm{CiOSC}$. The results indicated that 
CiMYB42 is a transcriptional activator in the limonoid metabolic network.

\section{Methods}

Plant materials and sampling

In April 2017, fresh healthy citrus leaves were collected at three different stages (Y1, Y2 and Y3) from different accessions in the National Citrus Germplasm Repository located in the Citrus Research Institute of the Chinese Academy of Agricultural Sciences (CRIC), in Beibei, Chongqing, China. Nine citrus accessions from four species were used for gene expression analysis and limonoid determination (Table 1). The leaf samples from the three developmental stages are shown in Fig. S2.

\section{Extraction and quantification of limonin and nomilin}

The extraction and quantification of limonin and nomilin via HPLC were performed according to the method described by Sun et al. [13], and three biological replicates were performed. Because standard samples for most limonoid components are unavailable, only limonin and nomilin could be quantitatively analysed. Thus, the sum of nomilin and limonin was used to represent the limonoid content in this study.

\section{Bioinformation analysis of CiMYB42}

The amino acid sequence of CiMYB42 was obtained from the $C$. clementina genome database (https://phytozome. jgi.doe.gov/pz/portal.html\#!linfo?alias=Org_Cclementina).

The theoretical PI (isoelectric point) and MW (molecular weight) were predicted by EXPASY (https://web.expasy. org/compute_pi/). The simple modular architecture research tool (SMART) was used to confirm the domain sequence of CiMYB42. The protein sequences were subjected to BLAST searches against the Phytozome database to identify homologous sequences in other plant species. DNAMAN v.6.0 was used for multiple alignment analysis. The homologous sequences of Arabidopsis were used for the construction of the phylogenetic tree.

\section{DNA and RNA extraction, CDNA synthesis and relative expression analysis}

Genomic DNA was extracted via the CTAB method, and RNA was extracted using the RNAprep Pure Plant Kit following the manufacturer's instructions (Tiangen Biotech, Beijing, China). RNA $(1 \mu \mathrm{g})$ was reverse transcribed into cDNA using the PrimeScript 1st Strand cDNA Synthesis Kit with gDNA Eraser (Perfect Real Time) (Takara Biomedical Technology, Beijing, China). The detection of gene expression was performed by realtime qPCR using $1 \times$ iTaq $^{\text {Tix }}$ universal SYBR $^{\circ}$ Green Supermix (Bio-Rad). The primers used in these procedures are listed in supplementary Table. S1. Experiments were performed in three replications using the citrus
Actin gene for normalization, and relative expression levels were calculated using the $2^{-\Delta \Delta \mathrm{Ct}}$ method [52].

\section{Transformation and characterization of transgenic sweet orange}

The CDS of CiMYB42 was amplified and ligated into the Sac I/BamH I sites of pBI121CSH to obtain the overexpression vector. For the construction of the RNAi vector, a 462 bp fragment was PCR amplified and integrated into the pGBi vector. These two expression vectors present enormous advantages in the visualization of exogenous gene transformation because GFP-positive samples are easily illuminated with an ultraviolet lamp (Fig. 4e). The recombinant overexpression and RNAi vectors were transformed into epicotyl explants of Wanjincheng orange (Citrus sinensis (L.) Osbeck) as previously reported [53]. The transformants were selected on MS medium containing $50 \mathrm{mg} / \mathrm{ml}$ kanamycin. The positive shoots were grafted onto rootstocks of two-year-old Ziyang Xiangcheng $(C$. junos) after the detection of GFP fluorescence and the PCR amplification of genomic DNA. The integration and expression of the CiMYB42 gene in transformed shoots were further confirmed by RT-PCR analysis.

\section{Yeast one-hybrid $(\mathrm{Y} 1 \mathrm{H})$ assay}

The full-length CiMYB42 ORF was amplified and fused to the pGADT7 vector to create the prey for the assay. Sequences $(2 \mathrm{~kb})$ including the promoters of CiSQS (ciclev10028537m) and CiOSC (ciclev10010416m) were synthesized by Beijing Genomics Institute and inserted into the pAbAi vector as the bait. The primers used for vector construction are listed in Table. S1. Yeast onehybrid $(\mathrm{Y} 1 \mathrm{H})$ assays were performed using the Matchmaker Gold Yeast One-Hybrid System (Clontech, USA). pAbAi-SQS and pAbAi-OSC were linearized by the BbsI enzyme, and the minimal inhibitory concentration of aureobasidin A (AbA) was detected on SD/-Ura medium. The interactions between CiMYB42 and the promoters of CiSQS and CiOSC were subsequently tested by cotransformation of the linearized bait plasmid and prey plasmid into $\mathrm{Y} 1 \mathrm{H}$ Gold yeast competent cells on SD/-leu medium with the optimal AbA concentration.

\section{Electronic mobility shift assay (EMSA)}

A cDNA fragment of CiMYB42 was amplified using genespecific primers (Table S1) and inserted into the pMAL$\mathrm{C} 2 \mathrm{X}$ vector at the BamHI/PstI sites with a $6 \times \mathrm{His}$ tag. The recombinant pMAL-C2X-MYB42 plasmid was transformed into the E. coli Rosetta (DE3) strain. The pMALC2X-MYB42 protein was purified by the immobilized metal affinity chromatography method, and Ni Sepharose High Performance was used in this step according to the manufacturer's instructions (GE Healthcare, USA). Oligonucleotide probes were synthesized and biotin labelled by 
Wuhan GeneCreate Biological Engineering Co., Ltd. The binding activity between the protein and probes was detected in an electronic mobility shift assay (EMSA). The CiMYB42-bound DNA fragments were separated from the unbound fragments by polyacrylamide gel electrophoresis according to the instructions of the Chemiluminescent EMSA Kit (Beyotime Biotechnology, China).

\section{Statistical analysis}

Statistical analysis was performed using the SPSSV20.0 statistical package. Significant differences were subjected to Duncan's test. $P<0.05$ was considered significant. Correlation analysis was conducted via Pearson's correlation analysis.

\section{Supplementary information}

Supplementary information accompanies this paper at https://doi.org/10. 1186/s12870-020-02475-4.

Additional file 1: Table S1. Primers and probes used in this study.

Additional file 2: Figure S1. Schematic diagram of limonoid biosynthesis. The abbreviations of the compounds and enzymes are as follows: IPP, isopentenyl diphosphate; DMAPP, dimethylallyl diphosphate; FPP, farnesyl diphosphate; FPPS, FPP synthase; SQS, squalene synthase; SQE, squalene epoxidase, OSC, oxidosuqlene cyclase; UDPG, UDPglycosyltransferase; NG, nomilin-glucopyranoside; LG, limoninglucopyranoside.

Additional file 3: Figure S2. Developmental stages of the leaf samples used in this study.

Additional file 4: Figure S3. The TF-binding cis-elements of the CISQS and CIOSC promoters.

Additional file 5: Figure S4. The minimal AbA inhibitory concentration of the bait vector. (a) pAbAi-SQS; (b) pAbAi-OSC.

Additional file 6: Figure S5. Expression and purification of the CiMYB42 protein. (a) The recombinant CiMYB42 protein expressed in Rosetta (DE3); (b) The supernatant and deposition of the CiMYB42 protein were examined by SDS-PAGE after ultrasonication; (c) SDS-PAGE analysis of recombinant and purified CiMYB42 protein; Lane M: protein ladder (116.0/66.2/45.0/35.0/25.0/18.4/14.4 kD); Lane 1: uninduced Rosetta (DE3) carrying PMAL-C2X; Lane 2: Rosetta (DE3) carrying PMAL-C2X induced by 1 mM IPTG; Lane 3: supernatant of the CiMYB42 protein; Lane 4: precipitate of the CiMYB42 protein; Lane 5: purified CiMYB42 protein; Lane 6: purified CiMYB42 protein. The molecular weight of recombinant CiMYB42 protein is $72.21 \mathrm{kD}$. The gel image were cropped because original image includes some of other samples not related to this study.

Additional file 7 Figure S6. The original gel image of Figure S5. (a) The original image of Figure S5a; (b) The original image of Figure S5b; (c) The original image of Figure $\mathrm{S} 5 \mathrm{c}$.

Additional file 8 Figure $\mathbf{S 7}$. The original gel image of EMSA.

\section{Abbreviations}

IPP: Isopentenyl diphosphate;" DMAPP: Dimethylallyl diphosphate;" MVA: Mevalonate; MEP: Methylerythritol phosphate; FPP: Farnesyl diphosphate; SQS: Squalene synthase; SQE: Squalene epoxidase; OSC: Oxidosqualene cyclases; TF: Transcription factor; CDS: Coding sequence; ORF: Open reading frame; JC: Beibei 447 orange; NHE: Newhall navel orange; GXSTY: Guangxi Shatian pummelo; ZSXLMY: Early Siam pummelo; ST: Morita Unshu Mandarin; GC: Miyagawa Wase Mandarin; WCZPG: Wangcang Zoupigan mandarin; HPJG: Huapi kumquat; RAJG: Rongan kumquat; SD: Synthetic dropout; Ura: Uracil; Leu: Leucine; AbA: Aureobasidin A; EMSA: Electronic mobility shift assay.

\section{Acknowledgements}

The authors greatly acknowledge Aihong Peng at the Citrus Research Institute of Southwest University for providing useful supervision of sweet orange transformation.

\section{Authors' contributions}

$\mathrm{PZ}$ and X-CZ designed the experiments; PZ conducted the experiments and data analysis and wrote the first draft of the manuscript; $X-C Z$ and $X Y$ edited the manuscript. X-FL provided the expression vector and scientific guidance on the genetic transformation of Wanjincheng orange; XY provided analytical tools and performed bioinformatic analysis. F-SW helped with sample preparation and limonoid quantification. J-HL and DJ contributed useful suggestions for the yeast one-hybrid assay. W-XS conducted the GPCR analysis; DJ conducted sample selection and provided valuable discussions. All authors have read and approved the final manuscript.

\section{Funding}

This study was supported by the "Double World-classes" Development Plan of Southwest University, providing the research platform for the experiment. We thank the National Key R\&D Program of China (2019YFD1001401-02), the Ministry of Education Double First-class Discipline Construction Project "Breeding and Application of New Crop Varieties and Germplasms", the Earmarked Fund for the Chongqing Special \& Economic Agriculture Research System on Late Maturation Citrus and the Earmarked Fund for the China Agriculture Research System (CARS-26) for the financial support of the HPLC, $\mathrm{Y} 1 \mathrm{H}$ and EMSA analyses. The Foundation for Talent/Doctors of Southwest University (SWU117038) and the Chongqing Research Project of Foundations and Frontiers (cstc2018jcyjAX0400) provided us financial support for the sequencing analysis and data analysis.

\section{Availability of data and materials}

All data analysed in this study are included in this published article and its supplementary files. The sequences of the genes used and analysed in current study are available at Phytozome database (https://phytozome.jgi. doe.gov/pz/portal.html) and the accession numbers are as follows: ciclev10021695m, orange1.1g024441m, AT4G12350.1, GRMZM2G104551_T01, Potri.003G114100.1, Manes.02G034300.1, Thecc1EG016054t1,

Gorai.003G168100.1, MDP0000682032, Glyma.01G211500.1, GSVIVT01004317001, mrna32268.1-v1.0-hybrid, Medtr4g102380.1, evm.model.supercontig_3.300, ciclev10028537m and ciclev10010416m. The plant materials are available from the corresponding author upon reasonable request.

Ethics approval and consent to participate

Not applicable.

\section{Consent for publication}

Not applicable.

\section{Competing interests}

The authors declare that they have no competing interests.

Received: 9 March 2020 Accepted: 27 May 2020

Published online: 03 June 2020

\section{References}

1. Gualdani R, Cavalluzzi M, Lentini G, Habtemariam S. The chemistry and pharmacology of citrus limonoids. Molecules. 2016;21(11):1530.

2. Qin S, Lv C, Wang Q, Zheng Z, Sun X, Tang M, Deng F. Extraction, identification, and antioxidant property evaluation of limonin from pummelo seeds. Animal Nutrition. 2018;4(3):281-7.

3. Roy A, Saraf S. Limonoids: overview of significant bioactive triterpenes distributed in plants kingdom. Biol Pharm Bull. 2006;29(2):191-201.

4. Vásquez-Rivera A, Chicaiza-Finley D, Hoyos RA, Orozco-Sánchez F. Production of limonoids with insect antifeedant activity in a two-stage bioreactor process with cell suspension culture of Azadirachta indica. Appl Biochem Biotech. 2015;177(2):334-45.

5. Fan X, Lin P, Lu X, Zi J. A new spiro-type limonoid from Azadirachta indica a. Juss Tetrahedron Lett. 2019;60(16):1158-60.

6. Maneerat W, Laphookhieo S, Koysomboon S, Chantrapromma K. Antimalarial, antimycobacterial and cytotoxic limonoids from Chisocheton siamensis. Phytomedicine. 2008;15(12):1130-4. 
7. Chen J, Fan X, Zhu J, Song L, Li Z, Lin F, Yu R, Xu H, Zi J. Limonoids from seeds of Azadirachta indica a. Juss. And their cytotoxic activity. Acta Pharm Sin B. 2018;8(4):639-44.

8. Chidambara Murthy KN, Jayaprakasha GK, Patil BS. Obacunone and obacunone glucoside inhibit human colon cancer (sw480) cells by the induction of apoptosis. Food Chem Toxicol. 2011;49(7):1616-25.

9. El-Readi MZ, Hamdan D, Farrag N, El-Shazly A, Wink M. Inhibition of pglycoprotein activity by limonin and other secondary metabolites from citrus species in human colon and leukaemia cell lines. Eur J Pharmacol. 2010;626(2-3):139-45.

10. Yan Y, Yuan C, Di Y, Huang T, Fan Y, Ma Y, Zhang J, Hao X. Limonoids from Munronia henryi and their anti-tobacco mosaic virus activity. Fitoterapia. 2015;107:29-35.

11. Yu J, Wang G, Han Y, Wu Y, Wainberg MA, Yue J. Limonoids with anti-hiv activity from Cipadessa cinerascens. J Nat Prod. 2015;78(6):1243-52.

12. Hu J, Song Y, Mao X, Wang Z, Zhao Q. Limonoids isolated from Toona sinensis and their radical scavenging, anti-inflammatory and cytotoxic activities. J Funct Foods. 2016;20:1-9.

13. Sun C, Chen K, Chen Y, Chen Q. Contents and antioxidant capacity of limonin and nomilin in different tissues of citrus fruit of four cultivars during fruit growth and maturation. Food Chem. 2005;93(4):599-605.

14. Bauri AK, Foro S, Nhu QND. Limonin isolated from the seeds of Citrus limetta (indian sweet lemon). IUCrData. 2016:1:2.

15. Li S, Wang Z, Ding F, Sun D, Ma Z, Cheng Y, Xu J. Content changes of bitter compounds in 'Guogin no.1' Satsuma mandarin (Citrus unshiu Marc.) during fruit development of consecutive 3 seasons. Food Chem. 2014;145:963-9.

16. Vranova E, Coman D, Gruissem W. Network analysis of the mva and mep pathways for isoprenoid synthesis. Annu Rev Plant Biol. 2013;64:665-700.

17. Xu R, Fazio GC, Matsuda SPT. On the origins of triterpenoid skeletal diversity. Phytochemistry. 2004;65(3):261-91.

18. Pandreka A, Dandekar DS, Haldar S, Uttara V, Vijayshree SG, Mulani FA, Aarthy T, Thulasiram HV. Triterpenoid profiling and functional characterization of the initial genes involved in isoprenoid biosynthesis in neem (Azadirachta indica). BMC Plant Biol. 2015;15:1.

19. Fu J, Liu G, Yang M, Wang X, Chen X, Chen F, Yang Y. Isolation and functional analysis of squalene synthase gene in tea plant Camellia sinensis. Plant Physiol Bioch. 2019;142:53-8.

20. Wang F, Wang M, Liu X, Xu Y, Zhu S, Shen W, Zhao X. Identification of putative genes involved in limonoids biosynthesis in citrus by comparative transcriptomic analysis. Front Plant Sci. 2017;8.

21. Han JY, Jo HJ, Kwon EK, Choi YE. Cloning and characterization of oxidosqualene cyclases involved in taraxasterol, taraxerol and bauerenol triterpene biosynthesis in Taraxacum coreanum. Plant Cell Physiol. 2019; 60(7):1595-603.

22. Jiang D, Rong Q, Chen Y, Yuan Q, Shen Y, Guo J, Yang Y, Zha L, Wu H, Huang L. Molecular cloning and functional analysis of squalene synthase (ss) in Panax notoginseng. Int J Biol Macromol. 2016;95:658.

23. Dong L, Pollier J, Bassard J, Ntallas G, Almeida A, Lazaridi E, Khakimov B, Arendt $P$, de Oliveira LS, Lota F, Goossens A, Michoux F, Bak S. Coexpression of squalene epoxidases with triterpene cyclases boosts production of triterpenoids in plants and yeast. Metab Eng. 2018:49:1-12.

24. Yang $Y$, Ge F, Sun Y, Liu D, Chen C. Strengthening triterpene saponins biosynthesis by over-expression of farnesyl pyrophosphate synthase gene and RNA interference of cycloartenol synthase gene in Panax notoginseng cells. Molecules. 2017;22(4):581.

25. Zheng X, Luo X, Ye G, Chen Y, Ji X, Wen L, Xu Y, Xu H, Zhan R, Chen W. Characterisation of two oxidosqualene cyclases responsible for triterpenoid biosynthesis in Ilex asprella. Int J Mol Sci. 2015;16(2):3564-78.

26. Zhong R, Lee $C$, Ye Z. Evolutionary conservation of the transcriptional network regulating secondary cell wall biosynthesis. Trends Plant Sci. 2010; 15(11):625-32

27. Shang Y, Ma Y, Zhou Y, Zhang H, Duan L, Chen H, Zeng J, Zhou Q, Wang S, Gu W, Liu M, Ren J, Gu X, Zhang S, Wang Y, Yasukawa K, Bouwmeester HJ, Qi X, Zhang Z, Lucas WJ, Huang S. Biosynthesis, regulation, and domestication bitterness in cucumber. Science. 2014;346(6213):1080-4

28. Mertens J, Pollier J, Vanden Bossche R, Lopez-Vidriero I, Franco-Zorrilla JM, Goossens A. The bHLH transcription factors TSAR1 and TSAR2 regulate triterpene saponin biosynthesis in Medicago truncatula. Plant Physiol. 2015; 170(1):194-210.

29. Tamura K, Yoshida K, Hiraoka Y, Sakaguchi D, Chikugo A, Mochida K, Kojoma M, Mitsuda N, Saito K, Muranaka T, Seki H. The basic helix-loop-helix transcription factor GUbHLH3 positively regulates soyasaponin biosynthetic genes in Glycyrrhiza uralensis. Plant Cell Physiol. 2018;59(4):783-96.

30. Singh AK, Kumar SR, Dwivedi V, Rai A, Pal S, Shasany AK, Nagegowda DA. A wrky transcription factor from Withania somnifera regulates triterpenoid withanolide accumulation and biotic stress tolerance through modulation of phytosterol and defense pathways. New Phytol. 2017;215(3):1115-31.

31. Mahjoub A, Hernould M, Joubès J, Decendit A, Mars M, Barrieu F, Hamdi S, Delrot S. Overexpression of a grapevine R2R3 MYB factor in tomato affects vegetative development, flower morphology and flavonoid and terpenoid metabolism. Plant Physiol Bioch. 2009;47(7):551-61.

32. Bedon F, Levasseur C, Grima-Pettenati J, Séguin A, MacKay J. Sequence analysis and functional characterization of the promoter of the Picea Glauca Cinnamyl Alcohol Dehydrogenase gene in transgenic white spruce plants. Plant Cell Rep. 2009;28(5):787-800.

33. Bedon F, Bomal C, Caron S, Levasseur C, Boyle B, Mansfield SD, Schmidt A, Gershenzon J, Grima-Pettenati J, Séguin A, Mackay J. Subgroup 4 R2R3 MYBs in conifer trees: gene family expansion and contribution to the isoprenoid- and flavonoid-oriented responses. J Exp Bot. 2010;61(14):3847-64.

34. Ding K, Pei T, Bai Z, Jia Y, Ma P, Liang Z. Smmyb36, a novel R2R3 MYB transcription factor, enhances tanshinone accumulation and decreases phenolic acid content in Salvia miltiorrhiza hairy roots. Sci Rep-Uk. 2017;7(1).

35. Reddy VA, Wang Q, Dhar N, Kumar N, Venkatesh PN, Rajan C, Panicker D, Sridhar V, Mao H, Sarojam R. Spearmint R2R3 MYB transcription factor msmyb negatively regulates monoterpene production and suppresses the expression of geranyl diphosphate synthase large subunit (msgpps.Lsu). Plant Biotechnol J. 2017;15(9):1105-19.

36. Matías-Hernández L, Jiang W, Yang K, Tang K, Brodelius PE, Pelaz S. AaMYB1 and its orthologue AtMYB61 affect terpene metabolism and trichome development in Artemisia annua and Arabidopsis thaliana. Plant J. 2017; 90(3):520-34.

37. Zhao K, Bartley LE. Comparative genomic analysis of the R2R3 MYB secondary cell wall regulators of arabidopsis, poplar, rice, maize, and switchgrass. BMC Plant Biol. 2014;14(1):135.

38. Kelemen Z, Sebastian A, Xu W, Grain D, Salsac F, Avon A, Berger N, Tran J, Dubreucq B, Lurin C, Lepiniec L, Contreras-Moreira B, Dubos C. Analysis of the DNA-binding activities of the arabidopsis R2R3 MYB transcription factor family by one-hybrid experiments in yeast. PLoS One. 2015;10(10):e141044.

39. Jia N, Liu J, Sun Y, Tan P, Cao H, Xie Y, Wen B, Gu T, Liu J, Li M, Huang Y, Lu J, Jin N, Sun L, Xin F, Fan B. Citrus sinensis MYB transcription factors CSMYB330 and CSMYB308 regulate fruit juice sac lignification through finetuning expression of the CS4CL1 gene. Plant Sci. 2018;277:334-43.

40. Xie R, Li Y, He S, Zheng Y, Yi S, LV Q, Deng L. Genome-wide analysis of citrus R2R3 MYB genes and their spatiotemporal expression under stresses and hormone treatments. PLoS One. 2014;9(12):e113971.

41. Dandekar DV, Jayaprakasha GK, Patil BS. Hydrotropic extraction of bioactive limonin from sour orange (Citrus aurantium L.) seeds. Food Chem. 2008; 109(3):515-20.

42. Wang F, Yu X, Liu X, Shen W, Zhu S, Zhao X. Temporal and spatial variations on accumulation of nomilin and limonin in the pummelos. Plant Physio Bioch. 2016;106:23-9.

43. Thimmappa R, Geisler K, Louveau T, O'Maille P, Osbourn A. Triterpene biosynthesis in plants. Annu Rev Plant Biol. 2014;65(1):225-57.

44. Gas-Pascual E, Berna A, Bach TJ, Schaller H. Plant oxidosqualene metabolism: cycloartenol synthase-dependent sterol biosynthesis in Nicotiana Benthamiana. PLoS One. 2014;9(10):e109156.

45. Suzuki M, Kamide Y, Nagata N, Seki H, Ohyama K, Kato H, Masuda K, Sato S, Kato T, Tabata S, Yoshida S, Muranaka T. Loss of function of 3-hydroxy-3methylglutaryl coenzyme a reductase $1 \mathrm{HMG} 1$ in arabidopsis leads to dwarfing, early senescence and male sterility, and reduced sterol levels. Plant J. 2004;37:750-61.

46. Hasegawa S, Herman Z. Biosynthesis of limonoids: conversion of deacetylnomilinate to nomilin in Citrus limon. Phytochemistry. 1986;25(11): 2523-4.

47. Cárdenas PD, Sonawane PD, Pollier J, Vanden Bossche R, Dewangan V, Weithorn E, Tal L, Meir S, Rogachev I, Malitsky S, Giri AP, Goossens A, Burdman S, Aharoni A. GAME9 regulates the biosynthesis of steroidal alkaloids and upstream isoprenoids in the plant mevalonate pathway. Nat Commun. 2016;7:1.

48. Yin J, Li X, Zhan Y, Li Y, Qu Z, Sun L, Wang S, Yang J, Xiao J. Cloning and expression of BpMYC4 and BpbHLH9 genes and the role of BpbHLH9 in triterpenoid synthesis in birch. BMC Plant Biol. 2017;17:1. 
49. Ma D, Reichelt M, Yoshida K, Gershenzon J, Constabel CP. Two R2R3 MYB proteins are broad repressors of flavonoid and phenylpropanoid metabolism in poplar. Plant J. 2018;96(5):949-65.

50. Xing C, Liu Y, Zhao L, Zhang S, Huang X. A novel MYB transcription factor regulates ascorbic acid synthesis and affects cold tolerance. Plant Cell Environ. 2019:42(3):832-45.

51. Zhong R, Lee C, Zhou J, McCarthy RL, Ye Z. A battery of transcription factors involved in the regulation of secondary cell wall biosynthesis in Arabidopsis. Plant Cell. 2008;20(10):2763-82.

52. Livak KJ, Schmittgen TD. Analysis of relative gene expression data using realtime quantitative PCR and the 2- ${ }^{\Delta \Delta C T}$ method. Methods. 2001;25(4):402-8.

53. Peng $A, X u L, H e Y$, Lei T, Yao L, Chen S, Zou X. Efficient production of marker-free transgenic 'Tarocco' blood orange (Citrus sinensis Osbeck) with enhanced resistance to citrus canker using a cre/loxp site-recombination system. Plant Cell Tissue Organ Culture (PCTOC). 2015;123(1):1-13.

\section{Publisher's Note}

Springer Nature remains neutral with regard to jurisdictional claims in published maps and institutional affiliations.

Ready to submit your research? Choose BMC and benefit from:

- fast, convenient online submission

- thorough peer review by experienced researchers in your field

- rapid publication on acceptance

- support for research data, including large and complex data types

- gold Open Access which fosters wider collaboration and increased citations

- maximum visibility for your research: over $100 \mathrm{M}$ website views per year

At BMC, research is always in progress.

Learn more biomedcentral.com/submissions 